ARTICLE

Received 7 Oct 2013 | Accepted 18 Feb 2014 | Published 17 Mar 2014

DOI: $10.1038 /$ ncomms4468

\title{
One-step pipetting and assembly of encoded chemical-laden microparticles for high-throughput multiplexed bioassays
}

Su Eun Chung ${ }^{1,2,3, \star}$, Jiyun Kim ${ }^{1,2,3, \star}$, Dong Yoon Oh ${ }^{1,3,4}$, Younghoon Song ${ }^{1,2,3}$, Sung Hoon Lee ${ }^{2}$, Seungki $\mathrm{Min}^{2}$ \& Sunghoon Kwon²,3

One quantitative liquid handling method in conventional assay processes is pipetting, which delivers a precise volume of one sample at a time. As this process becomes laborious and time-consuming as the number of samples increases, researchers in individual laboratories need a way to conduct large-scale assays in a reasonable amount of time and at an affordable cost. Here we report a novel handling technique of chemical substances termed 'partipetting', which allows the one-step pipetting of various chemical-laden hydrogels. We pipette and assemble various types of encoded chemical-laden microparticles in microwell arrays in parallel. The combination of this heterogeneous particle chip and a cell chip induces the release of the chemicals from the hydrogels and, eventually, the chemicals treat the targets. Based on bioassay applications using partipetting, we show its capability in large-scale bioassays, without the need for high-throughput bioassay resources, owing to a reduction in the assay costs and time.

\footnotetext{
${ }^{1}$ Center for Nanoparticle Research, Institute for Basic Science (IBS), Seoul National University, Gwanakro, Gwanak-gu, Seoul 151-744, South Korea. ${ }^{2}$ Department of Electrical and Computer Engineering, Seoul National University, Gwanakro, Gwanak-gu, Seoul 151-744, South Korea. ${ }^{3}$ Inter-university Semiconductor Research Center, Seoul National University, Gwanakro, Gwanak-gu, Seoul 151-744, South Korea. ${ }^{4}$ Interdisciplinary program of Bioengineering, Seoul National University, Gwanakro 1, Gwanak-gu, Seoul 151-744, South Korea. * These authors contributed equally to this work. Correspondence and requests for materials should be addressed to S.K. (email: skwon@snu.ac.kr).
} 
A dvances in a wide range of assay technologies have accelerated the discovery process for novel biochemical and pharmaceutical substances ${ }^{1-4}$. Prescreened candidate compounds and combinations of compounds may have high diagnostic or therapeutic potential for specific applications; thus, identifying these candidates in an efficient manner has assumed great importance in the biomedical industry. Unfortunately, the discovery and verification processes that necessitate the investigation of chemical molecules addressing a broad range of requirements involve considerable reagent costs and reaction times. Thus, these processes tend to be conducted only by large pharmaceutical companies and a few resourceful research institutes. Therefore, individual laboratories, which cannot afford exorbitant assay costs and high-priced equipment, have increased the demand for new strategies to provide more equal access to large-scale bioassays with reasonable costs and time requirements.

Quantitative liquid handling is one of the essential operations in the assay process and critically influences assay cost and time. Manual pipetting is predominantly used to deliver a precise volume of a sample to conventional microtiter plates, and researchers in individual laboratories usually deliver the reagents to the assay platform by manually pipetting one liquid sample at a time. This routine process becomes very laborious and time consuming as the number of test samples increases. If the researcher wants to design a quantitative assay investigating many types of chemicals, then the same number of separate pipetting events will be required for each type of chemical. Additionally, for a conventional pipette, the minimum handling volume of the liquid is limited to the microlitre level, which causes a significant, costly waste of targets, compounds and reagents.

The pipetting technique has been automated to make this process less labour-intensive. Automated liquid handlers dispense a selected quantity of reagent into a designated container in a rapid manner and have led to significant advances in dispensing throughput over the past few years compared with manual pipetting. However, in addition to the equipment expense and complicated operation, the dramatic increase in throughput directly results in a dramatic increase in reagent costs because automated systems consume the same amount of reagents as does the manual pipetting method. The reagent cost is the main obstacle for many small laboratories that want to expand their assays to a large scale. In terms of reaction volumes, microfluidics-based assays can be used to implement numerous reactions in nanolitre-sized reactors, which is much smaller than in pipetting-based assays ${ }^{5-9}$. Unfortunately, implementing a microfluidic device in large-scale multiplexed assays remains challenging, mainly because of the increased complexity of the interface for material delivery, for example, plumbing tubes and pumps.

An important question is how to provide an opportunity for researchers in individual laboratories to conduct large-scale bioassays in a reasonable amount of time and, more importantly, at an affordable cost. To that end, a new strategy should facilitate reaction volume reduction and parallel and heterogeneous liquid handling. In this article, we present a multiplexed bioassay method based on a novel handling procedure of chemical substances and name this process 'partipetting'. This technique represents one-step pipetting together with directed assembly of encoded chemical-laden hydrogels. Here the hydrogel microparticles, which have two-dimensional (2D) graphical barcodes and can hold sub-nanolitre volumes of chemicals, are first prepared for each chemical. Then different encoded hydrogel particles loaded with chemicals are mixed to form a pooled ECM library. This step facilitates the simultaneous handling of many encoded chemical-laden microparticles (ECMs), despite being mixed in the same vial, by virtue of the particles' individual identifying characteristics. To illustrate the potential of our technique, we performed multiplexed and combinatorial cytotoxicity assays of osteosarcoma cells (U2OS) with various anticancer drugs and confirmed that the partipetting-based assay method is compatible with a conventional assay technique. To perform this type of testing, we simply pipette various ECMs into the assay platform. The ECMs are then subjected to heterogeneous directed assembly in separate microwells. The unique code on the particles facilitates the identification of each ECM, even though numerous ECMs are randomly assembled in the microwells. Face-to-face attachment of this heterogeneous particle chip to a target cell chip induces the release of chemical substances from the encoded hydrogels and a subsequent reaction with the cells. With only a single pipetting event on a single chip, we can conduct $\sim 5,500$ simultaneous cytotoxicity assays, which is equivalent to an individual assay with 57 conventional 96-well microtiter plates. The real value of the partipetting technique is in the feasibility of large-scale quantitative bioassays in individual laboratories, without any aid from a high-priced automated liquid handling station. Therefore, we anticipate that our technique will enable individual researchers to perform multiplexed and highthroughput bioassays with greatly reduced processing time, reagent costs and labour.

\section{Results}

ECMs and partipetting. Partipetting, as we define it, is a single pipetting operation for the simultaneous directed assembly of many different ECMs in separate reaction wells in a microfabricated microwell plate, thus implying 'pipetting of encoded particles'. This method reduces not only the number of processes needed for chemical substance loading, owing to the simultaneous handling of numerous microparticles, but also the assay cost, owing to a dramatic reduction in the total volume of handed chemicals (Supplementary Table 1). Figure 1 shows the concept of 'partipetting'. The partipetting-based bioassay starts with the fabrication of ECMs, which involves the photopolymerization of hydrogel microparticles (Supplementary Fig. 1), followed by loading of chemical substances. In other words, the ECMs are photopolymerized hydrogels with a unique code representing the type of chemical that is physically entrapped within the polymer matrix. The hydrogel microparticles can hold a sub-nanolitre volume of chemicals, which is much less than in other delivery methods of chemicals ${ }^{10-13}$, and these hydrogels are individually identifiable by their $2 \mathrm{D}$ graphical barcodes ${ }^{14-19}$. To prepare the ECM library, each chemical is loaded into a correspondingly encoded hydrogel, and these ECMs are stored in a shrunken and dried form. The ECM library is simply generated by mixing selected ECMs into a single pool, as shown in Fig. 1a-c. This library is maintained in an oil solution, such as a solution containing mineral or silicon oil, until a release cue (Supplementary Fig. 2). When a ECM touches a liquid buffer, such as cell culture medium, chemical substances can immediately diffuse from the hydrogel matrix to the outer buffer solution ${ }^{20,21}$ (Fig. 1a inset).

Generally, researchers in individual laboratories deliver chemicals to a platform by manually pipetting samples one at a time. Thus, they rarely have the opportunity to perform largescale assays because of limited resources. However, in the partipetting process, the ECMs are pipetted from a pooled ECM library into a microwell plate, and with a single pipetting event, these microparticles are assembled in many separate microwells to form a variable particle chip. We have adopted a scraper-assisted assembly method to deliver the microparticles to the microwells and to remove excess microparticles remaining on the substrate 22,23 . 
a

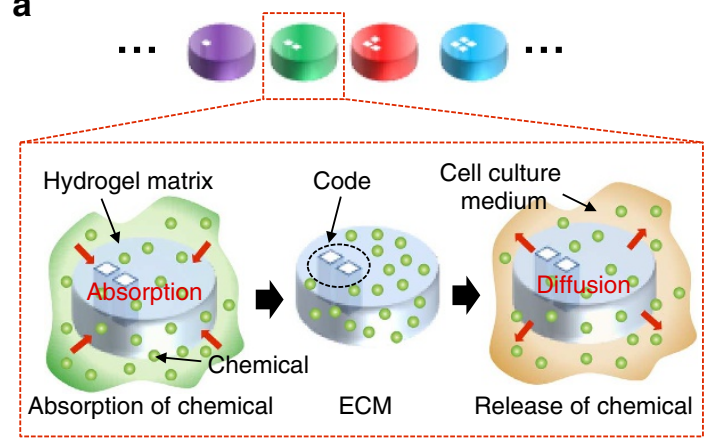

b

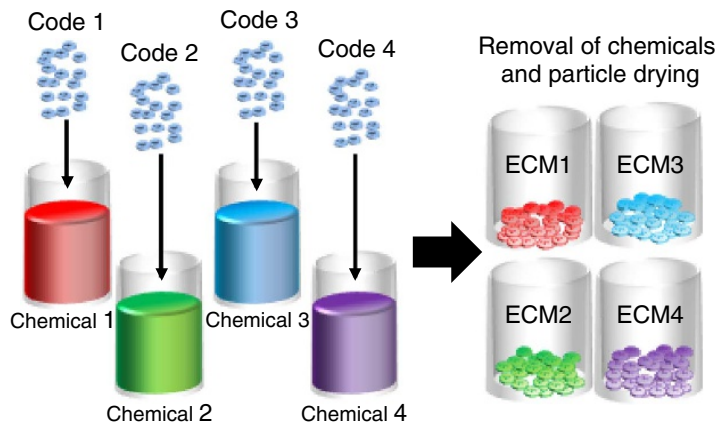

C
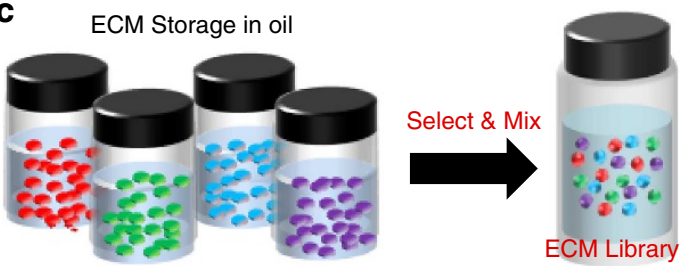

d
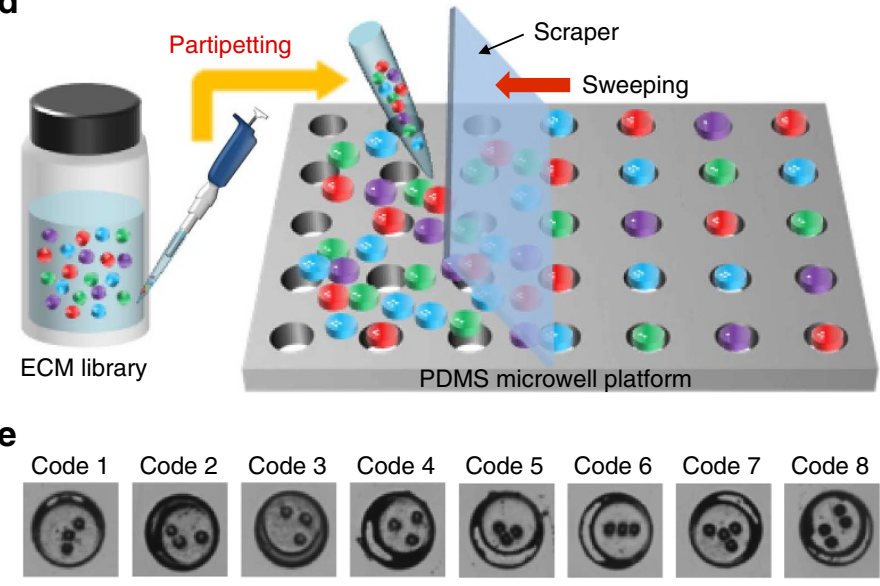

f

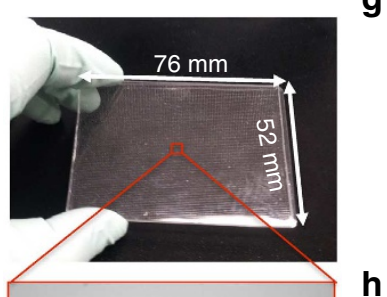

g

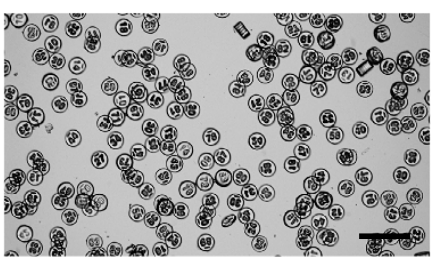

h
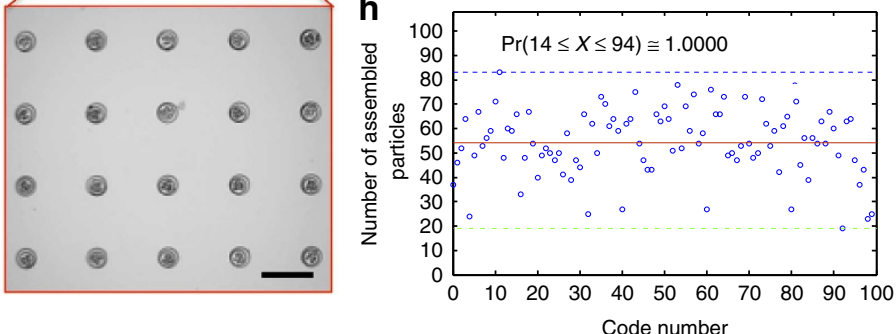

Figure 1 | The concept of partipetting as a cell-based bioassay platform. (a) Encoded chemical-laden microparticles (ECMs). Each code represents a type of chemical absorbed in the hydrogel matrix. The ECMs selected for the bioassay are mixed together in a single pool, forming an ECM library. The inset (red dashed box) shows absorption of chemicals into the hydrogel matrix and release of chemicals from the ECM. The chemicals absorbed into the hydrogels are released into the buffer by diffusion when the hydrogel comes into contact with either the cell culture medium or buffer solution. (b) ECM preparation. Initially, chemicals are absorbed into the hydrogel matrix through nonspecific absorption. After the absorption of chemicals, excess solution is removed, and the ECMs are dried. (c) ECM library preparation. Dried ECMs are stored in mineral oil, and selected ECMs are mixed together in a single pool for pipetting. (d) The concept of partipetting. Combining the words 'particles' and 'pipetting' results in the term 'partipetting'. As the term suggests, encoded microparticles in oil are pipetted into a microwell platform with carrier fluid and assembled in microfabricated wells by sweeping the substrate using a scraper. A single pipetting of various ECMs results in heterogeneity of the assay platform. (e) ECMs with eight different codes in microwells. Each ECM $(180 \mu \mathrm{m}$ in diameter) is positioned in a single well (200 $\mu \mathrm{m}$ in diameter). The code represents the chemical absorbed into the particle. (f) Bright-field microscopy of encoded microparticles assembled in microwells. (g) The number-coded microparticles used in Fig. 1f, from codes 00-99. (h) Code distribution of the assembled microparticles. Initially, particles were loaded into a microwell platform, with identical quantities per code, to empirically determine whether partipetting produces an even code distribution after assembly. The number of assembled particles per code is between a minimum of 19 and a maximum 83, with a mean value of 54 (green dotted line, blue dotted line and red line in Fig. $2 \mathrm{~h}$, respectively). Scale bars, $500 \mu \mathrm{m}$.

The composition of a particle chip depends on the random distribution of encoded microparticles on the substrate. Thus, the code distribution of microparticles should be determined to guarantee the multiplexing capability of this platform. We hypothesized that the assembled microparticles in our particle chip have a code distribution analogous to that of the loaded particles in the microwell plate and verified this concept experimentally by partipetting 100,000 microparticles with 100 different codes in a $76 \times 52 \mathrm{~mm}$-sized chip with 7,523 effective microwells (Fig. 1f). The microparticles were loaded into a microwell plate, with identical quantities of each code, or $\sim 1,000$ particles per code (Fig. 1g), and we counted the number of particles assembled in the particle chip to analyse the code distribution via partipetting. As shown in the code distribution displayed in Fig. 1h, we had a minimum of 19 microparticles, a maximum of 83 particles and an average of 54 assembled particles per code in the particle chip. The assembly yield was $72 \%$, which corresponded to a total of 5,416 microwells filled with particles. We estimated the theoretical probability of an assembled particle being in the chip per code to be 1 in 14-94 wells, using a binomial distribution (Supplementary Methods). Therefore, the counted number of assembled particles across all codes fits in the estimated range. This result signifies that the assembled ratio of the microparticles with 100 different codes was nearly analogous to the loaded ratio, as we expected. 
Chip preparation and assay procedure. In a cell-based assay using the partipetting approach, we prepare a particle chip with assembled ECMs and a separate cell chip with seeded target cells to prevent premature release of chemical substances. These two chips are combined face-to-face to permit the release of chemicals and subsequent exposure to the cells only when the user wants to perform an assay, as shown in Fig. 2a. After the combined chips have been incubated in an appropriate environment for the assay, the chips are separated, and the assay results are analysed.

For particle chip preparation, ECMs are partipetted into a $30 \times 30 \mathrm{~mm}$ microfabricated polydimethylsiloxane (PDMS) microwell platform. Partipetting involves the delivery of various ECMs from a single pool to the well platform, followed by the directed assembly of the particles in the microwells. During the partipetting process, oil is used as a carrier liquid to assemble the particles smoothly and to prevent the cross-contamination of chemicals (Supplementary Fig. 3). When a lubricating solution is not used, the dried hydrogels tend to be easily broken by the scraper during the assembly process. Figure $2 \mathrm{~d}$ shows cell staining with dye-laden microparticles assembled in a microwell platform. Although all of the various particles are randomly assembled in the microwell plate, each particle can be easily identified by its shape code. Note that a single ECM fits into a single microwell because the size and height of the microparticle are slightly smaller than those of a microwell. Each microparticle has a diameter of $180 \mu \mathrm{m}$ and a thickness of $60 \mu \mathrm{m}$, resulting in a subnanolitre volume capacity to contain chemicals, and a microwell has a diameter of $200 \mu \mathrm{m}$ and a depth of $80 \mu \mathrm{m}$. This dimension matching prevents the unwanted stacking of microparticles, thus minimizing the overloading of microparticles in a single well. As a result, because the partipetting-based approach reduces the laborious repetition of heterogeneous chemical substance introduction into the microwell plates by exploiting the ECM library, various types of chemicals can be handled within few pipetting events.

The preparation of the cell chip involves dispensing $4 \mathrm{ml}$ of cells in culture medium into the microwell plate $\left(10^{5}\right.$ cells per $\mathrm{ml}$ ), and the dispersed target cells then settle on the bottom of the a

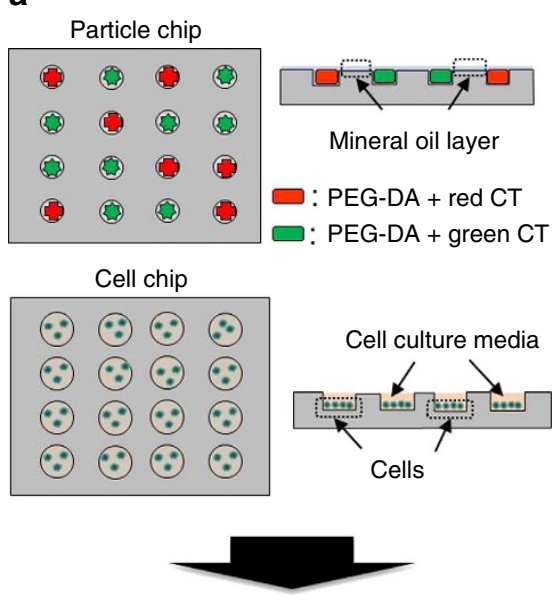

Align and combine two chips face-to-face

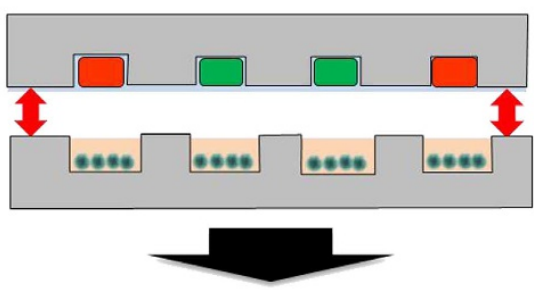

Release of chemicals by diffusion

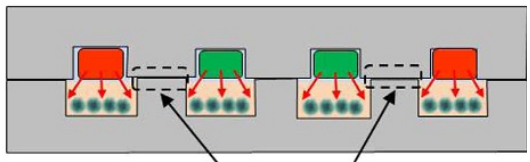

Oil layer for well isolation b

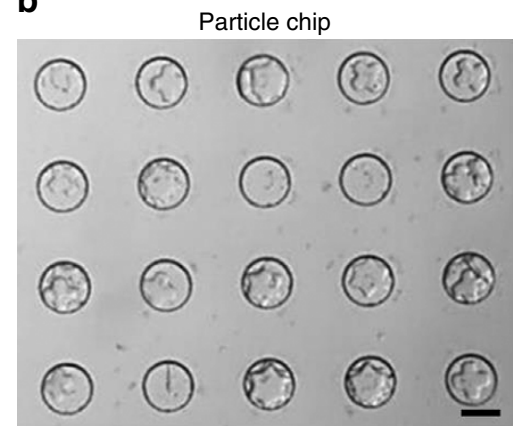

C

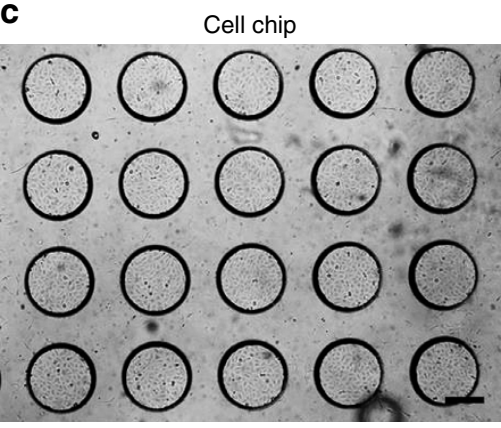

d

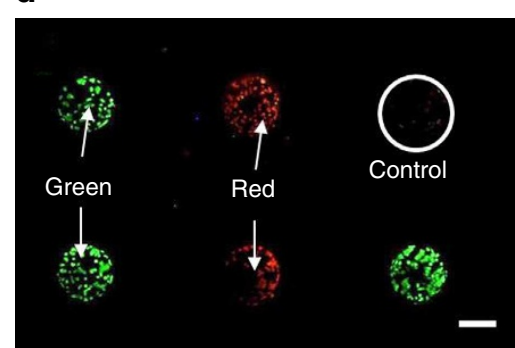

Figure 2 | Partipetting-based heterogeneous cell staining. (a) Schematic diagram of the chemical substance release process. Particle chip and cell chip for heterogeneous cell staining. Cell-staining dyes (red and green CT) are loaded into the photopolymerized poly (ethylene glycol) diacrylate (PEG-DA) particles. The particle chip and the cell chip are aligned and combined face-to-face to facilitate the release of chemical substances. Target cells in the cell chip react with the chemicals released from the particle chip. The chemicals are released by diffusion through the contact surface between an ECM and cell culture medium. The mineral oil layer from the particle chip prevents the cross-contamination of chemicals (Supplementary Fig. 6). (b) Image of a particle chip. Encoded microparticles are assembled in a particle chip. Each microwell in a particle chip has a diameter of $200 \mu \mathrm{m}$ and a depth of $60 \mu \mathrm{m}$. (c) Image of a cell chip. A cell chip is filled with seeded cells. Each microwell has a diameter of $500 \mu \mathrm{m}$ and a depth of $300 \mu \mathrm{m}$. (d) Fluorescence image of cells heterogeneously stained via partipetting. Scale bars, $300 \mu \mathrm{m}$. 
wells by gravity. After settling of the cells for $30 \mathrm{~min}$, we sweep over the surface of the plate using a cell scraper to eliminate excess cells from the substrate ${ }^{23,24}$. Figure $2 c$ shows the target cells seeded in microwells after overnight incubation, with an average of $167 \pm 47$ cells per well. Each microwell in the cell chip has a diameter of $500 \mu \mathrm{m}$ and a depth of $300 \mu \mathrm{m}$, which is larger than the particle, providing sufficient culture medium to the cells.

The prepared particle chip and cell chip are eventually combined in the release step of chemical substances, forming a sandwich structure ${ }^{13,25,26}$. During this process, the chemicalladen microparticles come into contact with the cell culture medium, and diffusion-mediated release of chemicals from the hydrogel matrix begins immediately after the two chips are combined (Supplementary Figs 4 and 5). During the release process of chemical substances, it is important to ensure that each well is completely isolated from the other wells to prevent the cross-contamination of chemicals. The oil used in the partipetting process functions as an isolating material, as shown in Fig. 2a. The oil layer on the contact surface between the cell chip and the particle chip prevents the mixing of chemicals between wells, thereby allowing independent and isolated release of chemicals (Supplementary Fig. 6).

In the proof-of-concept experiment shown in Fig. 2, two types of the cell-staining dye CellTracker (CT) were loaded into shapeencoded hydrogels to show heterogeneous cell staining via the partipetting method. The star-shaped microparticle contained a green-staining dye, and the circle-shaped microparticle contained a red-staining dye. Target cells seeded in the microwells were incubated overnight, and, as shown in Fig. 2d, the cells in each microwell were successfully labelled with the corresponding cellstaining dye.

Multiplexed cytotoxicity assay. We conducted a multiplexed cytotoxicity assay to illustrate the potential of the partipettingbased bioassay. In particular, we performed a cell viability assay to observe the activity of anticancer drugs against U2OS human osteosarcoma cells. The ECM library included eight anticancer drugs: camptothecin (CPT), sodium salicylate (SS), paclitaxel (PTX), auranofin (AF), PKF118-310 (PKF), and three combinatorial drugs containing different concentrations of CPT and SS.

Before conducting the cytotoxicity test, an evaluation of the exact concentration of anticancer drugs released from the ECMs is required because the chemicals are loaded into the hydrogels via absorption during the ECM fabrication process and released once in contact with cell culture medium. The molecular concentration of the released anticancer drugs was measured using a UV spectrophotometer (Supplementary Fig. 7). The light absorbance of several known concentrations of each anticancer drug was measured to generate a reference calibration curve, after which the absorbance of the anticancer drugs released from the ECMs into deionized (DI) water was measured to determine the released anticancer drug concentrations. Table 1 shows the loading and released concentrations of eight different anticancer drugs into and from the ECMs, respectively. The drug release rate is defined as the percentage ratio of the released concentration to the loading concentration of an anticancer drug, and this rate depends on the molecular weight of each anticancer drug. For example, PTX ( $\mathrm{MW}=853.91)$ released $3.3 \%$ of its loading concentration, and CPT $(\mathrm{MW}=348.4)$ released $13.6 \%$ of its loading amount. The final concentrations of the anticancer drugs delivered to the target cells were identical to the released concentrations from the ECMs.

After the drug release profiles had been characterized using a UV spectrometer, we prepared the particle chip with eight types of ECMs and the cell chip with U2OS cells, combined these two chips face-to-face and allowed them to react for $24 \mathrm{~h}$ in an incubator. Note that the culture medium in the cell chip should be changed to serum-free culture medium before combining the two chips to enhance the drug efficacy against the cells. After the incubation, the particle chip was removed, and the cell chip was treated with an FITC Annexin V Apoptosis Detection Kit to assess cell death by apoptosis or necrosis. FITC-annexin V binds to phosphatidylserine, which is translocated to the outer cell membrane, producing green fluorescence. Based on annexin $\mathrm{V}$ binding, we can identify apoptotic cells, that is, the cells that have reacted with the anticancer drugs. In addition, propidium iodide (PI), a membrane-impermeable red fluorescent DNA-staining dye, labels necrotic cells that have damaged membranes. Thus, the assay labels the cells in the following way: viable cells are annexin $^{-} / \mathrm{PI}^{-}$, early apoptotic cells are annexin ${ }^{+} / \mathrm{PI}^{-}$and late apoptotic or necrotic cells are annexin ${ }^{+} / \mathrm{PI}^{+}$. Figure $3 a$ shows microwell images of cells treated with drugs delivered via partipetting. The control group in this experiment consisted of cell-containing wells matched with wells containing no ECMs.

We quantified cell viability using the fluorescent images of anticancer drug-treated U2OS cancer cells. To count the total number of cells, including both viable and dead cells, we used a cell membrane-permeable DNA-staining dye (Hoechst 33342, Invitrogen). Figure $3 \mathrm{~b}$ shows DNA-stained U2OS cells in microwells, including both viable and dead cells, after $24 \mathrm{~h}$ of drug treatment. We determined the total number of cells to be 134 using image processing. Of a total of 134 cells, there were 30 green-fluorescent cells in the stage of apoptosis (Fig. 3c). In addition, there were 34 red-fluorescent cells, which represented damaged or late apoptotic cells, as shown in Fig. 3d.

To show the validity of the cytotoxicity assay result obtained by the partipetting-based method, we also measured the viability of the U2OS cells treated with drugs, using microtiter plate assays as reference data (Fig. 3e). The first reference was obtained by treating the cells with intact anticancer drugs as a control (blue bars in Fig. 3e). We dispensed $100 \mu \mathrm{l}$ of cells at $10^{5}$ cells per ml into each well in a 96-well microtiter plate and incubated the cells for $24 \mathrm{~h}$ to allow cell attachment. The cells in each well were then treated with $100 \mu$ l of anticancer drug solution dissolved in serum-free culture medium. The concentrations of drugs used in the experiments in the microtiter plate were identical to the

\begin{tabular}{|c|c|c|c|c|c|c|c|c|}
\hline & CPT & SS & PTX & AF & PKF & CPT:SS = 1:1 & CPT:SS = 1:2 & CPT:SS = 2:1 \\
\hline Molecular weight (MW) & 348.4 & 160.11 & 853.91 & 679.5 & 193.16 & & & \\
\hline Released concentration & $20.57 \mu \mathrm{M}$ & $20.8 \mathrm{mM}$ & $723 \mathrm{nM}$ & $779 \mathrm{nM}$ & $482 \mathrm{nM}$ & $\begin{array}{l}\text { CPT } 10.68 \mu \mathrm{M} \\
\text { SS } 10.2 \mathrm{mM}\end{array}$ & $\begin{array}{l}\text { CPT } 6.85 \mu \mathrm{M} \\
\text { SS } 6.79 \mathrm{mM}\end{array}$ & $\begin{array}{c}\text { CPT } 13.7 \mu \mathrm{M} \\
\text { SS } 3.4 \mathrm{mM}\end{array}$ \\
\hline
\end{tabular}


a

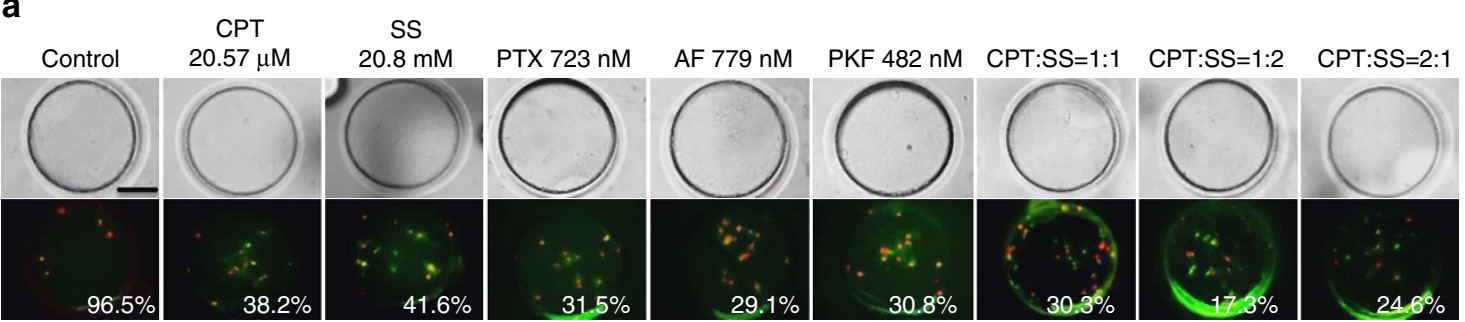

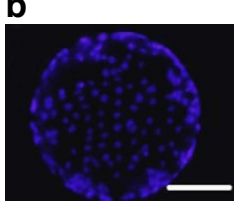

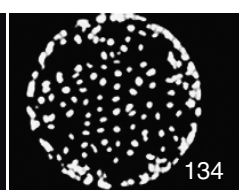

C
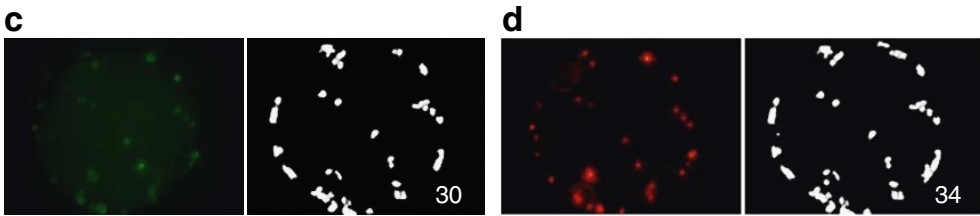

$\mathbf{e}$

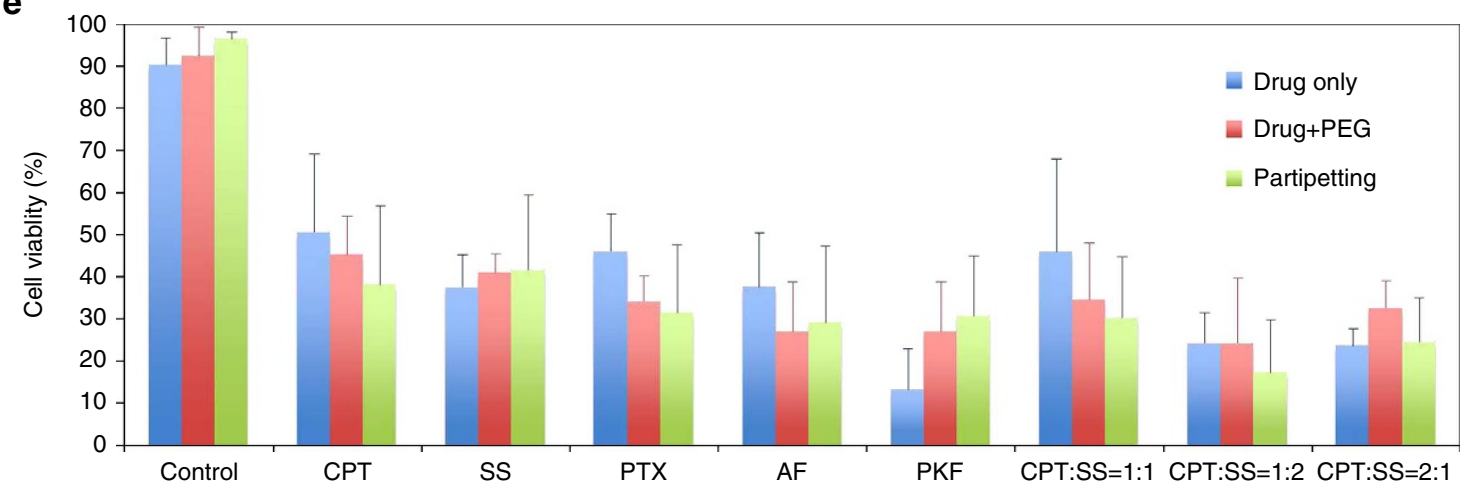

Figure 3 | A multiplexed cytotoxicity assay using cancer cells and eight anticancer drugs. (a) Multiplexed cytotoxicity assay by partipetting. Eight anticancer drugs (camptothecin (CPT), sodium salicylate (SS), paclitaxel (PTX), auranofin (AF), PKF118-310 (PKF), and three combinations of different concentrations of CPT and SS) released from ECMs were delivered to U2OS cancer cells seeded in a cell chip. The cells were treated with an FITC Annexin $\checkmark$ Apoptosis Detection Kit after $24 \mathrm{~h}$. The mean values of the measured cell viability in each condition are denoted in the fluorescence microscopy images. (b-d) Cell counting via image processing to measure viability. (b) Fluorescence image of total cells in a single microwell (DNA staining with Hoechst 33342) and binary image of counted cells. (c) Fluorescence image of apoptotic cells (FITC-annexin $\vee$ binding to externalized phosphatidylserine) and binary image of counted apoptotic cells. (d) Fluorescence image of necrotic cells (DNA staining by PI) and binary image of counted necrotic cells. (e) Comparison of cell viabilities obtained by a conventional technique and the partipetting technique. The blue bars in the graph represent the viabilities of cells treated with drugs alone in pipetting-based conventional microtiter plates, serving as reference data. The red bars represent the viabilities of cells treated with the same types and concentrations of anticancer drugs but in contact with photopolymerized PEG-DA microparticles in microtiter plates, used to confirm that the PEG-DA microparticles do not alter drug efficacy. The green bars represent cell viabilities obtained using the partipetting-based assay platform, and the data are within a maximum of $\pm 17 \%$ of the conventional assay data $(n \geq 10$; the values are means \pm s.d.).

Scale bars, $200 \mu \mathrm{m}$.

concentrations released from the ECMs, as listed in Table 1. In addition, it was necessary to prove that the drugs released from the polymeric hydrogels were still effective against cancer cells because all of the drugs were first loaded into poly(ethylene glycol) diacrylate (PEG-DA) particles and then delivered to the cells by diffusion. Therefore, the second reference was obtained by the same method, but with treatment of the cells with drugs that were kept in contact with photopolymerized PEG-DA microparticles for $24 \mathrm{~h}$ (red bars in Fig. 3e). As indicated by the green bars shown in Fig. 3e, the cell viabilities obtained by the partipetting-based assay were within $\pm 17 \%$ of the results of the conventional method. Therefore, the results obtained using the partipetting-based multiplexed bioassay platform provide an acceptable range of cell viabilities compared with those acquired using a conventional assay approach.

Scalable cell-based assay by partipetting. The particle and cell chips used in the previous proof-of-concept experiments have $\sim 400$ microwells in a $30 \times 30 \mathrm{~mm}$ chip (Figs 2 and 3). We can apply the merits of the partipetting technique to perform a largescale assay simply by increasing the size of the arrays. A multiplexed cytotoxicity assay was executed in a $76 \times 52 \mathrm{~mm}$ chip with $\sim 8,000$ microwells to confirm the viability of the scaled-up partipetting scheme using four anticancer drugs: CPT, SS, PTX and PKF (Fig. 4a). Figure 4b shows each ECM assembled in a microwell of the particle chip and the corresponding cell-based assay result in each case. The final concentrations of CPT, SS, PTX and PKF used to treat U2OS cells were $4.86 \mu \mathrm{M}, 33.46 \mathrm{mM}$, $842 \mathrm{nM}$ and $216 \mathrm{nM}$, respectively. The target cancer cells in the cell chip were reacted with the anticancer drugs released from each particle for $24 \mathrm{~h}$ and then stained using a FITC Annexin V Apoptosis Detection Kit to measure cell viability. We determined the cell viability of control and experimental groups treated with the same type and concentration of anticancer drug. Here, the control data, which represent a reference, were acquired using the conventional microtiter assay method, and the experimental data were obtained by the partipetting-based method. As observed in Fig. $4 \mathrm{c}$, the results of the partipetting-based assay were within $\pm 16 \%$ of the reference data. This finding implies that the 
a

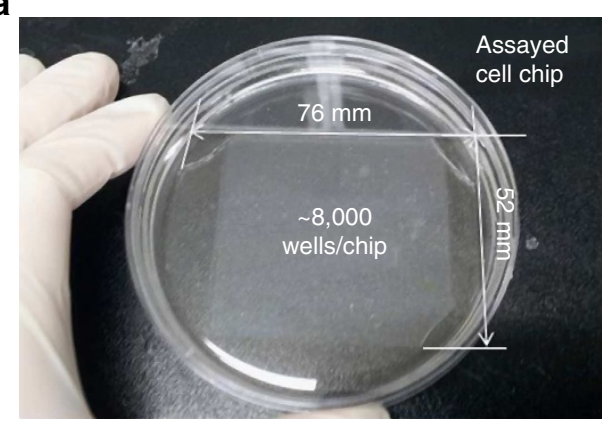

b

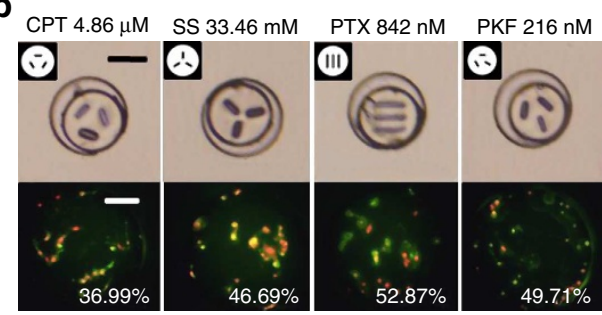

d

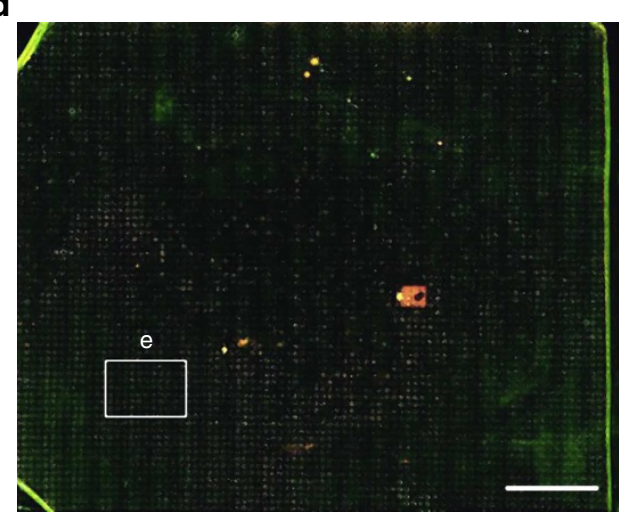

C
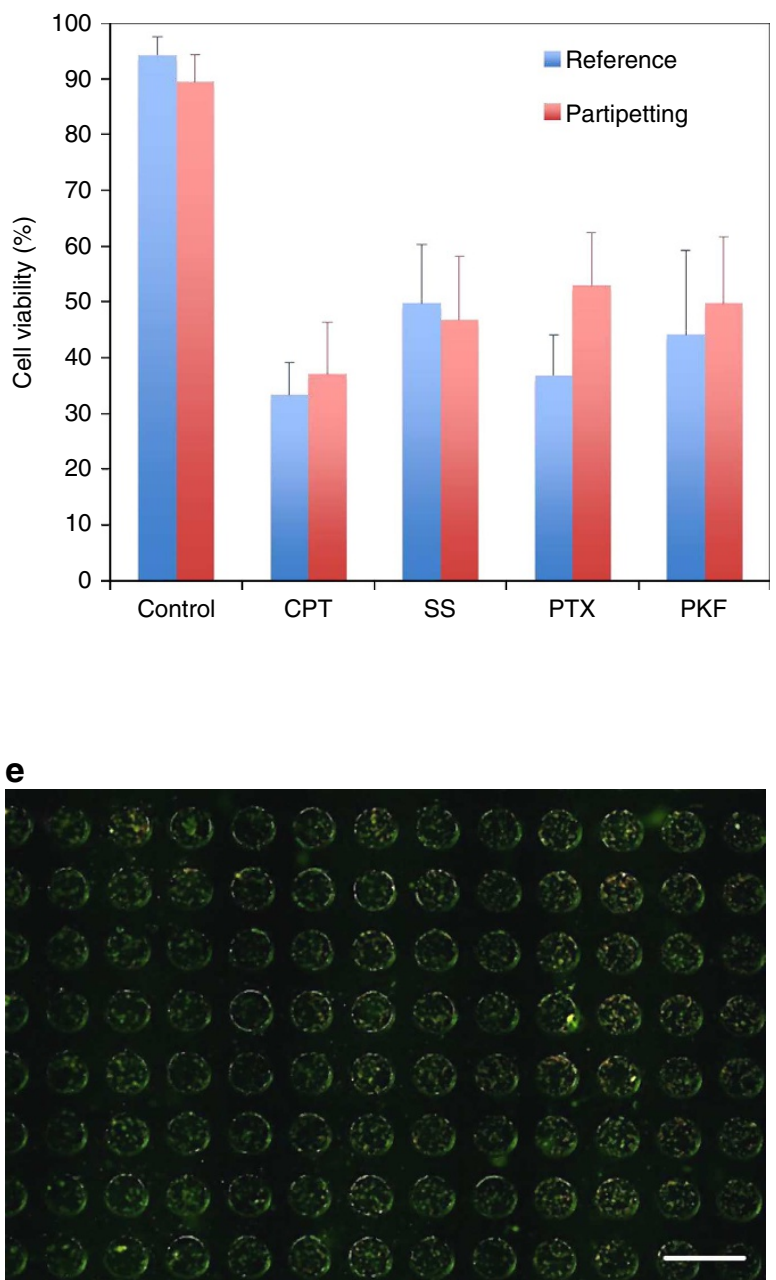

Figure 4 | A scalable multiplexed cytotoxicity assay by partipetting. (a) Photograph of the assayed cell chip. The size of both the particle and the cell chips was $76 \times 52 \mathrm{~mm}$, with $\sim 8,000$ microwells. (b) Four different drugs (CPT, SS, PTX and PKF) were loaded into microparticles and assembled in the microwells of a particle chip. The magnified view shows cells stained using an FITC Annexin V Apoptosis Detection Kit after $24 \mathrm{~h}$ of treatment with anticancer drug-loaded microparticles. Four different anticancer drugs, CPT, SS, PTX and PKF, were delivered to U2OS cancer cells using the scaled-up microwell platform. Scale bar, $100 \mu \mathrm{m}$. (c) Comparison between cell viabilities in conventional microtiter plates and in microwells treated using partipetting $(n \geq 23$; the error bars are means \pm s.d.) (d) Fluorescent microscopy image of the overall cell chip after staining using an apoptosis detection kit. Scale bar, $1 \mathrm{~cm}$. (e) Magnified view of the assayed cells in each section. Scale bar, $1 \mathrm{~mm}$.

partipetting-based assay provides results that are analogous to those obtained by a conventional method, even when the platform has been scaled up. Researchers in individual laboratories generally deliver each reagent to the assay platform by manually pipetting one at a time, but our assay method allows the delivery of 8,000 ECMs all at once, resulting in cost-effective and highthroughput bioassays.

Combinatorial cell-based assay by partipetting. The partipetting-based bioassay technique is not confined to a one-to-one matching scheme, implying that a single cell well is aligned with a single particle well, as demonstrated in Figs 2-4. More than one chemical can be delivered to the cells in each well simultaneously, thus enabling a combinatorial bioassay using the partipettingbased platform. Combinations of chemicals can be controlled according to the design of the placement of the microwell arrays in the particle chip. Figure 5a shows a particle chip whose combination consisted of $2,3,4$ or 5 microwells in a circular formation. Microparticles with 100 different numerical codes were used to illustrate various potential combinations of chemicals. Thus, many different combinations of chemical substances can be used to perform assays together in a single microwell, as shown in Fig. 5a.

We demonstrated heterogeneous cell staining to show the potential of combinatorial bioassays using the partipetting-based platform. Each independent combination set was aligned and combined face-to-face with a single cell well in the cell chip, enabling combinatorial delivery of chemical substances to the target cells. Here each combination set consisted of three particle wells in a triangular formation, and this set was matched with one cell well (Fig. 5b). For ECMs, we loaded three different cellstaining dyes (orange, green and blue CT) into encoded hydrogels to visualize combinations of various dyes. The labels ' 1 ', ' 2 ' and ' 3 ' in Fig. $5 \mathrm{c}$ represent orange, green and blue CT-loaded microparticles, respectively. Thus, when two chips were combined, the cells in a single microwell were simultaneously treated with three types of CT-loaded microparticles. Consequently, the cells could 
a

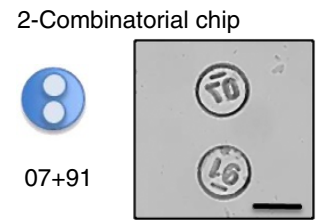

4-Combinatorial chip

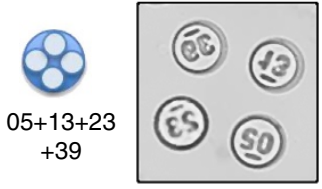

3-Combinatorial chip

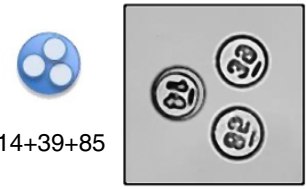

5-Combinatorial chip

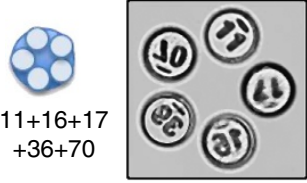

b

One assay set
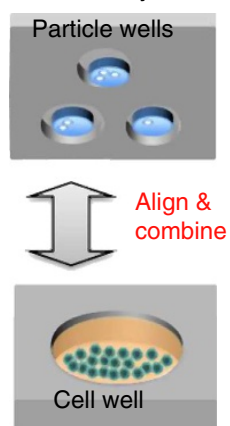

C

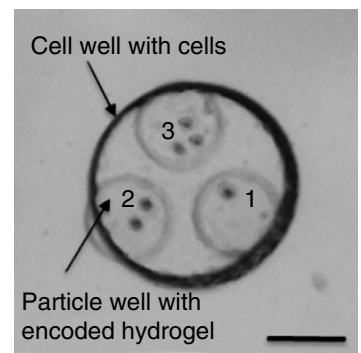

d

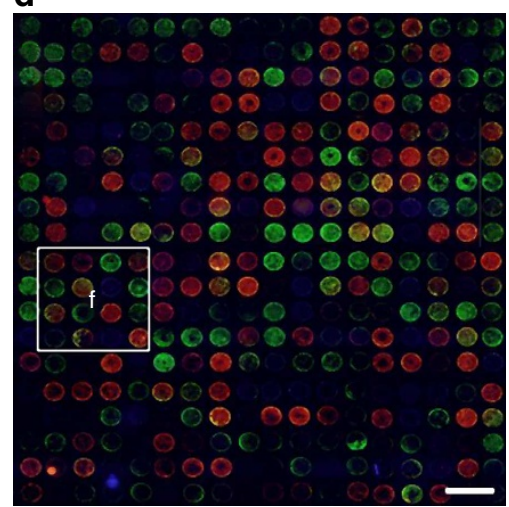

e

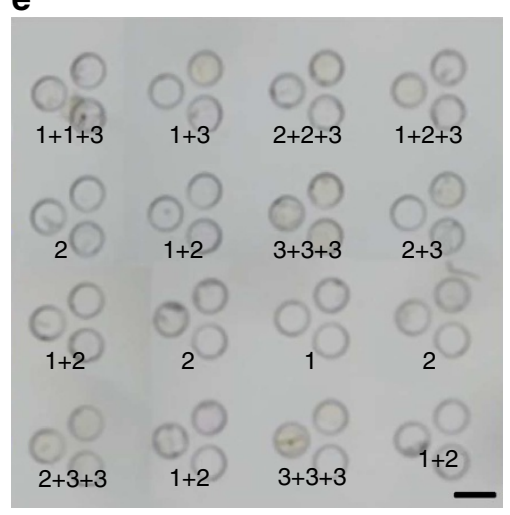

f

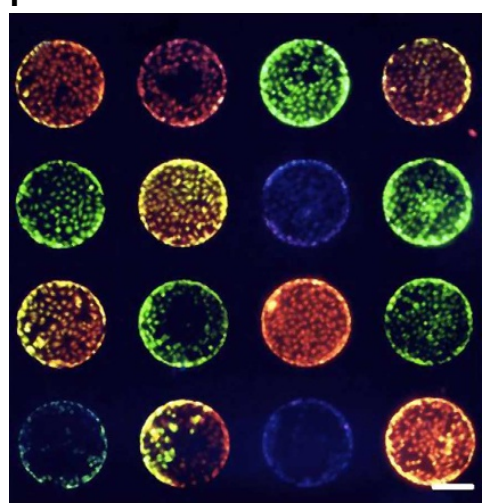

Figure 5 | Heterogeneous cell staining by combinatorial partipetting. (a) Various combinations of encoded microparticles in the particle chip. Microparticles with 100 different number codes were loaded and assembled in each particle chip, resulting in four different combinations of particles. Each combination of particles could be matched with a single microwell in the cell chip. Scale bar, $100 \mu \mathrm{m}$. (b) Schematic diagram of heterogeneous cell staining by three-chemical combinatorial partipetting. Three particle wells compose a single combination set in the particle chip, and this set is aligned and combined face-to-face with a single cell well of the cell chip, enabling various combinations of chemicals to be released in the target cell well. (c) Brightfield image of the combined particle and cell wells. Three different cell-staining dyes (orange, green and blue) are loaded into each encoded microparticle, and these ECMs are assembled in the particle chip for combinatorial partipetting. ' 1 ', ' 2 ' and ' 3 ' represent the orange, green and blue CT-loaded microparticles, respectively. In one combination set, the cells in a single microwell are treated with three ECMs at the same time. (d) Fluorescence image of the assayed cell chip after the staining process by combining the particle and cell chips with the assembled CT-loaded microparticles (orange, green and blue CT dyes). Scale bar, $1 \mathrm{~mm}$. (e,f) Matching of stained cells in the cell chip with each set of CT combinations in the particle chip. (e) Sectioned image from the particle chip. The numbers in the image represent each ECM from Fig. 5c. (f) Sectioned image from the cell chip in (d). The cells in each independent microwell of the cell chip were treated with three chemicals released from each combination set in the particle chip at the same time. Scale bars in Fig. $5 \mathbf{a}-\mathbf{c}, \mathbf{e}, \mathbf{f}, 200 \mu \mathrm{m}$.

be stained with a combination of orange, green and blue dyes, including duplicated color combinations.

Figure $5 \mathrm{~d}$ shows the cell chip assayed with the particle chip, composed of randomly assembled orange, green and blue CTloaded microparticles for combinatorial heterogeneous cellstaining experiments. The cells in each independent microwell were stained with a matching combination set, consisting of three CT-loaded microparticles. If two or three different CT dyes stained the same cells in the microwell, the fluorescence would merge the colors orange, green and blue, resulting in a color such as yellow, magenta, cyan or white. Figure $5 \mathrm{e}, \mathrm{f}$ shows paired sets of particle and cell chips, with combinatorial heterogeneous cell staining. As shown in the representative fluorescence images in Fig. 5f, the mixed fluorescence of the cells indicates that the cells were successfully stained by a combination of CT-loaded microparticles from the set shown in Fig. 5e.

In addition to combinatorial heterogeneous cell staining, we conducted a cytotoxicity assay using a three-chemical combinatorial particle chip. As shown in Fig. 6a, microparticles loaded with two different anticancer drugs were used, and each combination consisted of three particle wells in the particle chip, including microwells with no ECMs. This format allowed 10 different combinations of anticancer drugs in the particle chip (see concentrations in Table 2). Note that case 1 was classified as a control group because no ECMs were assembled in the combination set. Figure $6 \mathrm{~b}, \mathrm{c}$ shows a representative fluorescent image of cells stained using an FITC Annexin V Apoptosis Detection Kit after $24 \mathrm{~h}$ of treatment with combinatorial anticancer drugs. Here green and red fluorescence represents apoptotic and necrotic cells, respectively (Supplementary Figs 8 and 9).

To analyse cell viability in each drug-combination case, we needed to identify an address for each combination case in the particle chip and the target cells treated with the corresponding drug combination. For code mapping and data analysis, we developed an image-processing program using Python. We prepared overall images of a particle chip with randomly assembled ECMs and of an assayed cell chip, and the program then produced a map of codes for ECMs from the particle chip, a map of counted cells from the cell chip, and paired data from the particle chip and cell chip with calculated cell viabilities, as shown in Fig. $7 \mathrm{a}-\mathrm{d}$. We adopted the CellC algorithm to count viable and dead cells in fluorescent images ${ }^{27}$. The image processing required 
a Camptothecin (CPT) $4.86 \mu \mathrm{M}$ Sodium salicylate (SS) $33.46 \mathrm{mM}$

\begin{tabular}{|c|c|c|c|c|c|c|c|c|c|}
\hline Case 1 & Case 2 & Case 3 & Case 4 & Case 5 & Case 6 & Case 7 & Case 8 & Case 9 & Case 10 \\
\hline & (6) & (5) & (1) & 6 & $\begin{array}{l}(1) \\
\circ 6\end{array}$ & (2) 8 & $(8)$ & $\begin{array}{c}(9) \\
(6)\end{array}$ & $\begin{array}{c}(9) \\
(3)\end{array}$ \\
\hline
\end{tabular}

b

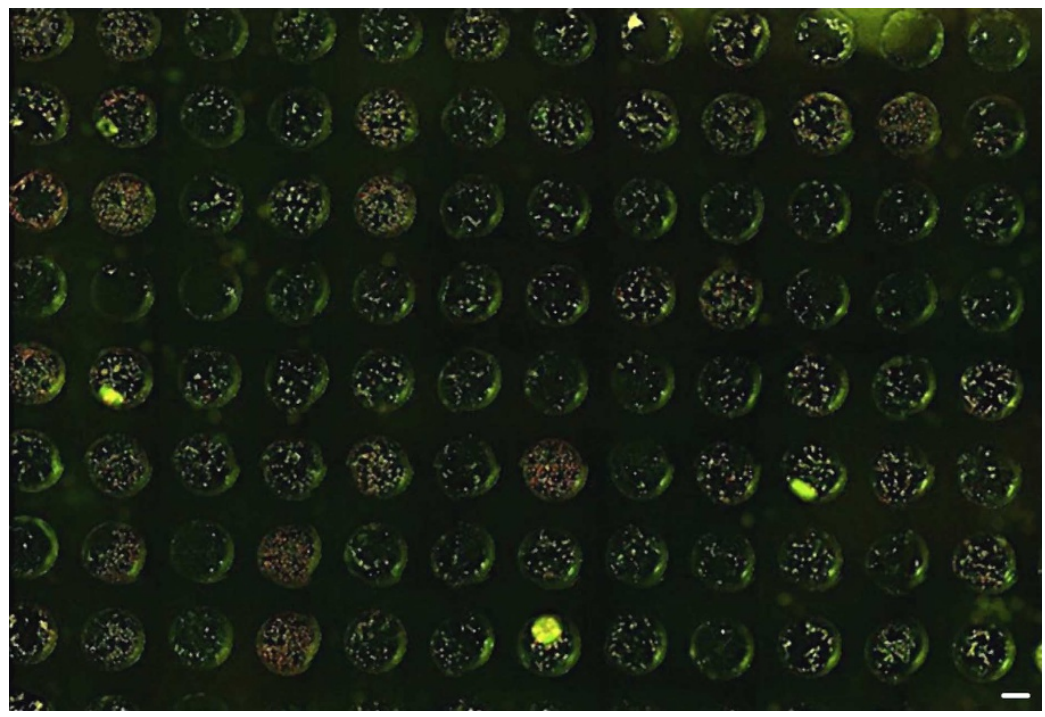

C

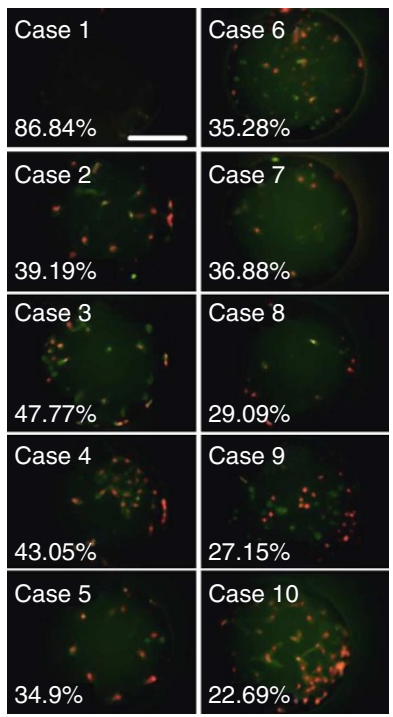

Figure 6 | A cytotoxicity test using a three-drug combinatorial particle chip. (a) Combination cases using two different anticancer drugs: camptothecin (CPT) and sodium salicylate (SS). A total 10 different combinations occurred in the particle chip, including microwells with no ECMs. Case 1 was a control group. (b) Fluorescence image of cells stained using an FITC Annexin V Apoptosis Detection Kit $24 \mathrm{~h}$ after combinatorial drug treatment in a three-drug combinatorial particle chip. (c) Each single cell well in the 10 combination cases. The mean measured value of cell viability in each case is denoted in the fluorescence images. Scale bars, $200 \mu \mathrm{m}$.

\begin{tabular}{|lccccc|}
\hline \multicolumn{6}{|c|}{ Table 2 I Concentrations of combinatorial anticancer drugs. } \\
Case & WELL1 & WELL2 & WELL3 & Conc. of CPT & Conc. of SS \\
\hline 1 & 0 & 0 & 0 & 0 & 0 \\
2 & 0 & 0 & CPT & $4.86 \mu \mathrm{M}$ & 0 \\
3 & 0 & 0 & SS & 0 & $33.46 \mathrm{mM}$ \\
4 & 0 & CPT & SS & $4.86 \mu \mathrm{M}$ & $33.46 \mathrm{mM}$ \\
5 & 0 & CPT & CPT & $9.72 \mu \mathrm{M}$ & 0 \\
6 & 0 & SS & SS & 0 & $66.92 \mathrm{mM}$ \\
7 & CPT & CPT & SS & $9.72 \mu \mathrm{M}$ & $33.46 \mathrm{mM}$ \\
8 & CPT & CPT & CPT & $14.58 \mu \mathrm{M}$ & 0 \\
9 & CPT & SS & SS & $4.86 \mu M$ & $66.92 \mathrm{mM}$ \\
10 & SS & SS & SS & 0 & $100.38 \mathrm{mM}$ \\
& & & & & \\
\hline
\end{tabular}

only a few minutes per chip, from decoding the assembled ECMs in the particle chip to mapping the cell viability data with the decoded drug-combination set.

Figure $7 \mathrm{a}, \mathrm{b}$ shows the decoding process of the drug combinations in the particle chip. We first obtained bright-field images of the particle chip using a $\times 4$ microscope objective view prior to the drug release step. In each $\times 4$ image of the particle chip, we had a maximum of 20 combinations of particle wells, as indicated by the green rectangles. All green rectangles were arranged and stitched to a single chip image by image processing to obtain an overall particle chip image. Each microwell with an ECM was denoted as a red circle, and the total particle chip image was then segmented into individual combinations composed of three microwells by red grids (Fig. 7a). After the recognition of microwells filled with ECMs, each combination set was decoded and classified by its respective case, which is defined in Fig. 6a. Figure $7 \mathrm{~b}$ shows all of the classified combination cases, from case 1 to case 10 , in a single particle chip. Here the red numbers represent the identified combination cases, and the blue squares indicate cases classified as unrecognizable. The classified cases of drug combinations could be modified when the drug combinations were identified incorrectly, and these cases are overlaid with both red and blue squares.

Similar to the particle chip segmentation, the cell chip image was also segmented into individual microwells by red grids to separately analyse cell viability in each microwell, as shown in Fig. 7c. The blue fluorescence indicates the total number of target cells, including both viable and dead cells that were stained with Hoechst 33342. A single cell chip had three independent images, blue, green and red fluorescence images, corresponding to total cells, apoptotic cells and necrotic cells, respectively, as previously explained in Fig. 3b. After segmentation of the cell chip into individual microwells, cells were recognized as pink dots and counted via image processing. Here the green squares are segments that were filtered to remove errors. Note that all of the blue, green and red fluorescence images were segmented by the same grid and counted to calculate cell viabilities according to each effective microwell (Supplementary Fig. 8). Cell viability is defined as the percentage of living cells in each microwell.

Finally, particle chip and cell chip images were matched to pair each combination case in the particle chip with the 

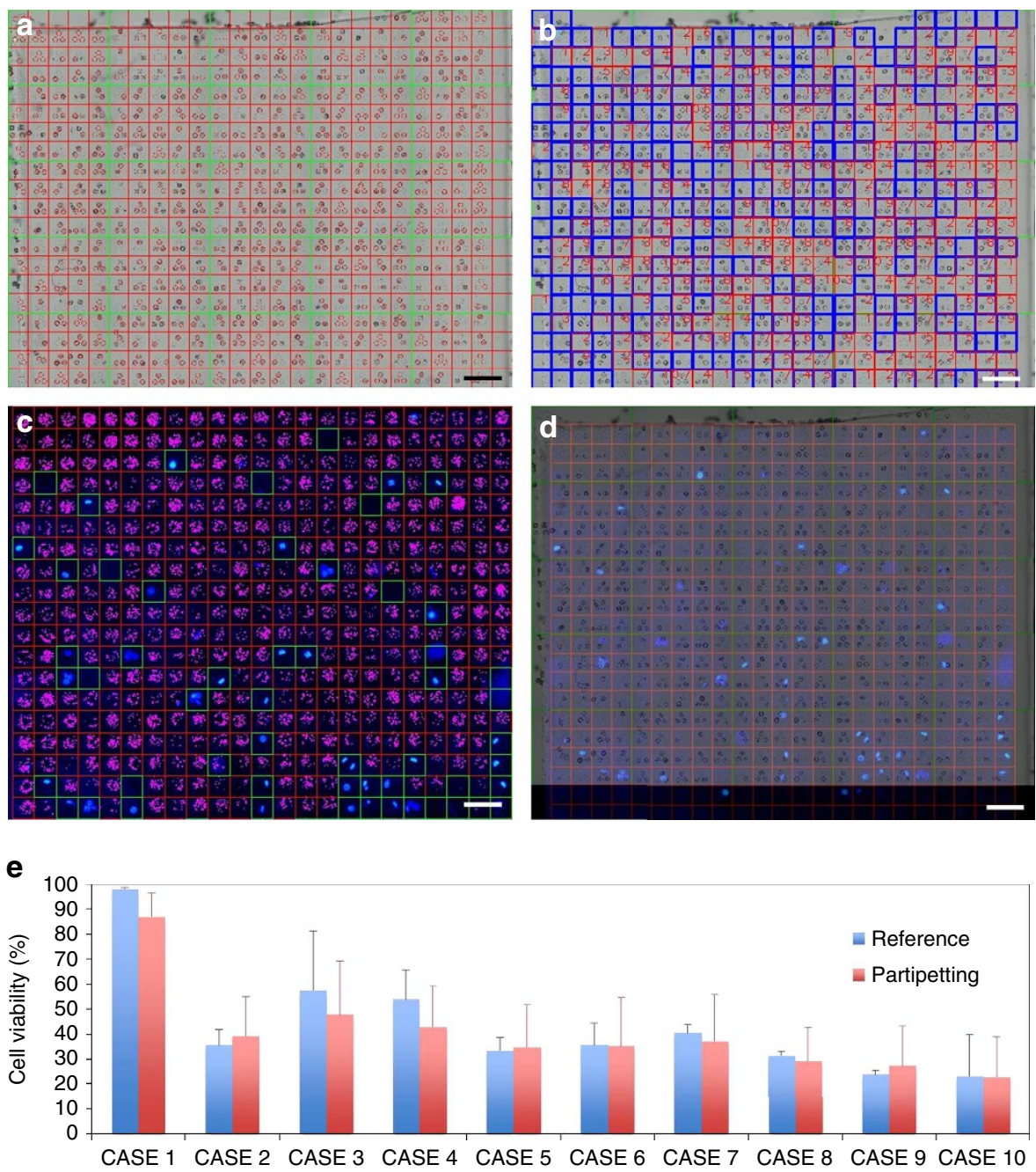

Figure 7 | Cell viability calculation from three-drug combinatorial partipetting data. (a) Particle chip with assembled ECMs for the combinatorial cytoxicity assay. Bright-field images ( $\times 4$ microscope objective view) were arranged and stitched to obtain an overall particle chip image. The chip image was segmented into each combination by image processing. Microwells with ECMs were identified in this step. Each red circle represents a microwell with a particle. (b) Classification of combination cases and case counting. Each combination is classified by case, from 1 to 10 . The red numbers are identified cases of each combination. The blue squares represent the cases that were filtered out as unrecognizable. Cases overlaid with both blue and red squares are modified cases that were initially identified incorrectly. (c) Cell chip segmented into individual microwells. To calculate the viabilities of cells treated with combinations of ECMs, cells were counted by image processing. The pink dots are cells recognized in each microwell. The green squares are segments that were filtered to remove errors. (d) Overlaid images of the particle and cell chips. Each combination case of the particle chip was paired with the corresponding microwells of the cell chip image. Scale bars, $1 \mathrm{~mm}$. (e) Comparison of cell viabilities between conventional microtiter plates using pipetting and a microwell platform using partipetting. The blue bars in the graph represent the viabilities of cells reacted with drugs in microtiter plates as a reference. The red bars represent cell viabilities measured in the three-drug combinatorial partipetting platform. The data are within a maximum of $\pm 10 \%$ of the conventional data ( $n \geq 43$; the values are means + s.d.)

corresponding microwells of the cell chip (Fig. 7d). After eliminating unrecognizable particle codes in the particle chip and errors in the cell chip, all cell viabilities were matched with each drug-combination case in the particle chip. The viabilities of the cells assayed by the combinatorial partipetting approach were then compared with those of cells assayed by the conventional method to demonstrate the reliability of the combinatorial partipetting-based assay result (Fig. 7e). The blue bars in the graph represent the apoptotic viabilities of cells treated with drugs alone in microtiter plates as a reference. The red bars are apoptotic cell viabilities obtained using the three-drug combinatorial partipetting method. The results were within $\pm 10 \%$ of those obtained by the conventional approach, showing the feasibility of a partipetting-based combinatorial cytotoxicity assay.

\section{Discussion}

In this study, we describe a partipetting-based bioassay platform using chemical-laden encoded microparticles. Partipetting potentially enables the handling of a large number of different chemicals in a single chip with a single operation and reagent cost reduction because of the sub-nanolitre volume of the hydrogel matrix. We demonstrated multiplexed and combinatorial cytotoxicity assays using cancer cell line and several anticancer drugs as simple applications of the partipetting-based approach. We believe that the partipetting technique is suitable for researchers who cannot afford an automated liquid handling system and huge reagent costs. Thus, compared with the manual pipetting method, our technique provides greater opportunities to perform large-scale quantitative assays in individual laboratories. Owing to its simplicity and flexibility, we envision that the 
proposed method will encourage innovation in the development of a wide range of various bioassays, including cell-based assays, enzymatic assays and protein-protein interaction studies, and a host of other future applications.

\section{Methods}

ECM fabrication. Encoded microparticles are fabricated in a high-throughput manner by an optofluidic maskless lithography (OFML) technique ${ }^{29}$, which enhances variations in graphical codes by dynamic masking via a digital micromirror device (DMD). The OFML system allows the encoding of hydrogels for drug identification by manipulating the DMD pattern during in situ photopolymerization (Supplementary Fig. 1). The code for each particle, which is a mask of DMD, is designed individually using the program Paint (Microsoft Windows, USA) and uploaded to the DMD software of the OFML system. The hydrogels are selectively photopolymerized by exposure to UV radiation at $100 \%$ intensity with a $0.1 \mathrm{~s}$ curing time through the $\times 10$ objective lens of the OFML system. Here each microparticle has a diameter of $180 \mu \mathrm{m}$ and a thickness of $60 \mu \mathrm{m}$. All of the fabricated hydrogel particles are first collected in ethyl alcohol $(\mathrm{EtOH})$ solution. To prevent the photoinitiator residue of uncured resin from inducing cell death inside the hydrogels, we repeat the washing with fresh EtOH solution to thoroughly wash the particles and then vortex mix or sonicate the hydrogels two to three times. We then let the particles sink to the bottom of the $\mathrm{EtOH}$ solution for a few minutes, after which we remove the EtOH. Next, the particles are placed in anti-adhesion dishes (that is, Falcon tube caps) or tubes. To completely remove any EtOH remaining around the microparticles, we dry them in a desiccator overnight or in an oven for $30 \mathrm{~min}$ at $50^{\circ} \mathrm{C}$.

Materials. We used poly(ethylene glycol) diacrylate (PEG-DA, $\mathrm{M}_{n}=700$; SigmaAldrich) with $5 \mathrm{wt} \%$ photoinitiator (2-hydroxy-2-methylpropiophenone $97 \%$, Sigma-Aldrich) to synthesize the polymeric structures. To demonstrate heterogeneous cell staining, we loaded 0.2 mM CT Red CMPTX, Orange CMTMR, Green CMFDA or Blue $\mathrm{CMF}_{2} \mathrm{HC}$ (Invitrogen) into the encoded microparticles. In the multiplexed cytotoxicity assays, we used anticancer drugs, including camptothecin (CPT, $\mathrm{M}_{n}=348.4$; Sigma-Aldrich), sodium salicylate (SS, $\mathrm{M}_{n}=160.11$; SigmaAldrich), paclitaxel (PTX, $\mathrm{M}_{n}=853.91$; Sigma-Aldrich), auranofin (AF, $\mathrm{M}_{n}=679.5$; Sigma-Aldrich) and PKF118-310 (PKF, $\mathrm{M}_{n}=193.16$; Sigma-Aldrich). For mixed drug treatments, CPT and SS were blended at ratios of 1:1, 1:2 and 2:1 to illustrate drug repositioning ${ }^{27,28}$. The microparticles were stored and handled in mineral oil (Sigma-Aldrich) or silicone oil (Sigma-Aldrich) during the partipetting process.

Measurement of released anticancer drug concentration. We prepared the set of 100,000 ECMs for each type of anticancer drug in duplicate. We used five anticancer drugs for the concentration measurements: CPT $(\mathrm{MW}=348.4)$, SS $(\mathrm{MW}=160.11), \mathrm{PKF}(\mathrm{MW}=193.16), \mathrm{PTX}(\mathrm{MW}=853.91)$ and AF $(\mathrm{MW}=853.91)$. During the partipetting process, a single ECM is exposed to target cells in an individual cell well. The dimensions of a microwell in the cell chip are $500 \mu \mathrm{m}$ (diameter) by $300 \mu \mathrm{m}$ (depth), giving the microwell a volume capacity of $59 \mathrm{nl}$. Therefore, for each set of drugs, 100,000 microparticles are reacted with $5.9 \mathrm{ml}$ of buffer (that is, DI water). After $24 \mathrm{~h}$ of drug release, we extracted the drugcontaining buffer to measure the drug concentrations. For the reference calibration curve, we measured the light absorbance of several known concentrations of each drug. The reference drugs used for the concentration measurements were drug solutions that were kept in contact with photopolymerized PEG-DA microparticles. We measured the light absorbance of unknown drug concentrations and fit the values to a reference calibration curve. In this way, we determined the final concentrations of drugs released into the buffer solution. Supplementary Fig. 7 shows the light absorbance graphs of each drug in DI water, including the reference and test data. In each graph, all the unknown and known concentrations of drugs are plotted. We compared the absorbance peaks at specific wavelengths to determine the concentration of each drug released into the buffer.

Drug release curve. To estimate a predictable drug concentration when a drug diffuses into medium, we generated a drug-release reference curve based on actual concentrations of released drugs. Supplementary Fig. $7 \mathrm{~b}$ shows an example: the drug-release reference curve of SS at 12 different drug-loading concentrations, which was used to assess the drug's release tendency. We first fabricated SS-loaded microparticles at 12 drug-loading concentrations: $0.2,1,2,10,20,50,100,200$ and $500 \mathrm{mM}$, and 1, 2 and $3 \mathrm{M}$. Note that the solubility of SS in water is up to $660 \mathrm{gl}^{-1}$, yielding a drug concentration of $4.1 \mathrm{M}$. In addition, the PEG-DA microparticles in this experiment were fabricated using $100 \%$ UV intensity in the OFML system. Each concentration set has 15,000 microparticles, and these particles were reacted with $885 \mu \mathrm{l}$ of DI water for $24 \mathrm{~h}$. We then measured the light absorbance of DI water to determine the released drug concentration. The concentration of drug released from ECMs is controllable within the linear range from $0.2 \mathrm{mM}$ to $1 \mathrm{M}$ for the loading concentration of SS. The concentration of released drug then saturates at a loading concentration of $\sim 1 \mathrm{M}$, a limit imposed by the maximum absorption capacity of the hydrogel matrix.
Cell culture. U2OS human osteosarcoma cells (KCLB, Korean Cell Line Bank) were cultured in McCoy's 5A culture medium with $1 \%$ penicillin-streptomycin and $10 \%$ fetal bovine serum at $37^{\circ} \mathrm{C}$ under $5 \% \mathrm{CO}_{2}$ and $95 \%$ atmospheric air. Cultured cells were detached from culture flasks by trypsinization using $0.25 \%$ trypsin and $0.13 \%$ EDTA in phosphate-buffered saline. The cells were centrifuged and dispersed in cell culture medium to produce a cell density of $\sim 10^{5}$ cells per ml. All of the PDMS microwell plates (both cell chips and particle chips) were first sterilized in an autoclave for $20 \mathrm{~min}$ at $120^{\circ} \mathrm{C}$, followed by oxygen plasma treatment for $1.5 \mathrm{~min}$. The disinfected microwell plates were treated with fibronectin (SigmaAldrich) for $45 \mathrm{~min}$ to enhance cell adhesion on and protein absorption to the PDMS microwell plates.

Release of chemicals and apoptosis assay. A cell chip with attached target cells in the microwells was aligned and placed face-to-face with the corresponding particle chip containing partipetted ECMs using a microscope. The assembled chips were then fixed with paper clips and incubated for $24 \mathrm{~h}$ to allow sufficient drug release by diffusion and apoptosis induction by the anticancer drugs. After reaction with the drugs, the cell chip was gently washed five times with phosphatebuffered saline and stained with an apoptosis-detecting solution (FITC Annexin V Apoptosis Detection Kit, BD Pharmingen) and membrane-permeable DNAstaining dye (Hoechst 33342, Invitrogen) for $20 \mathrm{~min}$ in an incubator.

\section{References}

1. Drews, J. Drug discovery: a historical perspective. Science 287, 1960-1964 (2000).

2. Dove, A. Drug screening-beyond the bottleneck. Nat. Biotechnol. 17, 859-863 (1999).

3. Carnero, A. High throughput screening in drug discovery. Clin. Transl. Oncol. 8, 482-490 (2006)

4. Imming, P., Sinning, C. \& Meyer, A. Drugs, their targets and the nature and number of drug targets. Nat. Rev. Drug Discov. 5, 821-834 (2006).

5. Thorsen, T., Maerkl, S. J. \& Quake, S. R. Microfluidic large-scale integration. Science 298, 580-584 (2002).

6. Whitesides, G. M. The origins and the future of microfluidics. Nature 442, 368-373 (2006).

7. Smith, C. Tools for drug discovery: tools of the trade. Nature 446, 219-222 (2007).

8. Dittrich, P. S. \& Manz, A. Lab-on-a-chip: microfluidics in drug discovery. Nat. Rev. Drug Discov. 5, 210-218 (2006).

9. Park, W., Han, S., Lee, H. \& Kwon, S. Free-floating amphiphilic picoliter droplet carriers for multiplexed liquid loading in a microfluidic channel. Microfluid. Nanofluid. 13, 511-518 (2012).

10. Khademhosseini, A. et al. A soft lithographic approach to fabricate patterned microfluidic channels. Anal. Chem. 76, 3675-3681 (2004).

11. Ramanan, R. M. K., Chellamuthu, P., Tang, L. \& Nguyen, K. T. Development of a temperature-sensitive composite hydrogel for drug delivery applications. Biotechnol. Prog. 22, 118-125 (2006).

12. Soppimath, K. S., Aminabhavi, T. M., Kulkarni, A. R. \& Rudzinski, W. E. Biodegradable polymeric nanoparticles as drug delivery devices. J. Controlled Release 70, 1-20 (2001).

13. Wu, J. et al. A sandwiched microarray platform for benchtop cell-based high throughput screening. Biomaterials 32, 841-848 (2011).

14. Lee, H., Kim, J., Kim, H., Kim, J. \& Kwon, S. Colour-barcoded magnetic microparticles for multiplexed bioassays. Nat. Mater. 9, 745-749 (2010).

15. Han, S. et al. Lithographically encoded polymer microtaggant using highcapacity and error-correctable QR code for anti-counterfeiting of drugs. Adv. Mater. 24, 5924-5929 (2012)

16. Kim, J., He, L., Song, Y., Yin, Y. \& Sunghoon, K. Lithographically compartmentalization of emulsion droplet templates for microparticles with multiple nanostructures compartments. Chem. Commun. 48, 6091-6093 (2012).

17. Braeckmans, K. et al. Encoding microcarriers by spatial selective photobleaching. Nat. Mater. 2, 169-173 (2003).

18. Dejneka, M. J. et al. Rare earth-doped glass microbarcodes. Proc. Natl Acad. Sci. USA 100, 389-393 (2003).

19. Pregibon, D. C., Toner, M. \& Doyle, P. S. Multifunctional encoded particles for high-throughput biomolecule analysis. Science 315, 1393-1396 (2007).

20. Lee, S. H., Heinz, A. J., Choi, S.-E., Park, W. \& Kwon, S. Polymer based chemical delivery to multichannel capillary patterned cells. Lab Chip 11, 605-608 (2011).

21. Lee, S. H. et al. Active guidance of 3D microstructures. Small 6, 2668-2672 (2010).

22. Ahn, J.-M., Kim, J. H., Kim, J. H. \& Gu, M. B. Randomly distributed arrays of optically coded functional microbeads for toxicity screening and monitoring. Lab Chip 10, 2695-2701 (2010).

23. Kang, L., Hancock, M. J., Brigham, M. D. \& Khademhosseini, A. Cell confinement in patterned nanoliter droplets in a microwell array by wiping. J. Biomed. Mater. Res. Part A 93A, 547-557 (2010). 
24. Moeller, H.-C., Mian, M. K., Shrivastava, S., Chung, B. G. \& Khademhosseini, A. A microwell array system for stem cell culture. Biomaterials 29, 752-763 (2008).

25. Du, W., Li, L., Nichols, K. P. \& Ismagilov, R. F. SlipChip. Lab Chip 9 2286-2292 (2009).

26. Zhou, X., Lau, L., Lam, W. W. L., Au, S. W. N. \& Zheng, B. Nanoliter dispensing method by degassed poly(dimethylsiloxane) microchannels and its application in protein crystallization. Anal. Chem. 79, 4924-4930 (2007)

27. Ashburn, T. T. \& Thor, K. B. Drug repositioning: identifying and developing new uses for existing drugs. Nat. Rev. Drug Discov. 3, 673-683 (2004).

28. Keith, C. T., Borisy, A. A. \& Stockwell, B. R. Multicomponent therapeutics for networked systems. Nat. Rev. Drug Discov. 4, 71-78 (2005).

29. Chung, S. E., Park, W., Park, H., Park, K. Y. N. \& Kwon, S. Optofluidic maskless lithography system for real-time synthesis of photopolymerized microstructures in microfluidic channels. Appl. Phys. Lett. 91, 041106 (2007).

\section{Acknowledgements}

This work was supported by the Institute for Basic Science (IBS) in Korea. This work was supported by a National Research Foundation of Korea (NRF) grant funded by the Korean government (MEST) (No. 2012-0009555, No. 2011-0028409, No. 2010-0017860, No. 2011-0030269, No. 2012M3A9B2030170). D.Y.O. acknowledge funding from Amorepacific R\&D Center. We thank Honggeun Oh and Sunghoon Kim of the
Biophotonics and Nanoengineering Laboratory, SNU, for their high-throughput fabrication of the encoded microparticles.

\section{Author contributions}

S.E.C., S.H.L. and S.K. designed the concept and initial experiments of the work. S.E.C., J.K. and D.Y.O. performed the experiments and analysis. Y.S. fabricated and provided the assay chip for the experiments. S.M. worked on the image processing for code reading and cell viability analysis.

\section{Additional information}

Supplementary Information accompanies this paper on http://www.nature.com/ naturecommunications

Competing financial interests: The authors declare no competing financial interests.

Reprints and permission information is available online at http://npg.nature.com/ reprintsandpermissions/

How to cite this article: Chung, S. E. et al. One-step pipetting and assembly of encoded chemical-laden microparticles for high-throughput multiplexed bioassays. Nat. Commun. 5:3468 doi: 10.1038/ncomms4468 (2014). 